Viharos, Zs. J.; Monostori, L.; Csongrádi, Z.; Realizing the digital factory: monitoring of complex production systems, Intelligent Manufacturing Systems, 2003, Elsevier, 2003, pp.

29-34.

\title{
REALIZING THE DIGITAL FACTORY: MONITORING OF COMPLEX PRODUCTION SYSTEMS
}

\author{
Zs. J. Viharos ${ }^{1}$, L. Monostori ${ }^{1}$, Z. Csongradi ${ }^{2}$ \\ ${ }^{1}$ Computer and Automation Research Institute, Hungarian Academy of Sciences, Budapest, Hungary \\ ${ }^{2}$ Machine Division, Electrical Development, GE Hungary, Budapest, Hungary
}

\begin{abstract}
Today's complex manufacturing systems operate in a changing environment rife with uncertainty. The performance of manufacturing companies ultimately hinges on their ability to rapidly adapt their production to current internal and external circumstances. On the base of a running national research and development project (NRDP) on digital enterprises and production networks, the paper illustrates how the concepts of intelligent manufacturing systems in view of digital enterprises and monitoring of complex production systems can contribute to the solution of the above problems. Copyright @ 2002 IFAC
\end{abstract}

Keywords: Digital systems, Factory automation, State monitoring, Simulators, Data handling systems

\section{INTRODUCTION}

Manufacturing systems in our epoch work in a fast changing environment full of uncertainties. Increasing complexity is another characteristic which shows up in production processes and systems and in enterprise structures as well (Merchant, M.E., 1998). The concept of the digital enterprise, i.e., the mapping of all the important elements of the enterprise processes by means of information technology tools gives a unique way of managing the above problems.

A Hungarian R\&D project was started to make all the important, production-related information available and manageable in a controlled, userdependent way by the efficient application of information and communication technologies (Monostori, 2002; Monostori et al., 2002). The development and application of intelligent decision support systems will help enterprises to cope with the problems of uncertainty and complexity, increase their efficiency, join in production networks and to improve the scope and quality of their customer relationship management. The project covers the following - partly overlapping - main directions to be treated in a comprehensive way:
- Management and scheduling of large-scale projects (Erdélyi, et al., 2002).

- Tele-presence and interactive multimedia (Haidegger, Popa, 2002).

- Monitoring of complex production structures (Viharos et al., 2002a).

These directions appoint the main parts, called clusters of the project, within the two-sided framework of project management and dissemination of results. One cluster incorporates three main work areas representing the continuous development starting with basic and applied research, followed by research and development (R\&D) assignments, and ending in the market-oriented demonstrations of the cluster results. Altogether eleven work packages are managed during the project life.

Clusters vary in the structure of the five participants and in their individual tasks. The main activity of the Budapest University of Technology and Economics and the Miskolc University is to do research in the first two clusters and they participate in the concerning $R \& D$ work as well. As a part of R\&D and demonstration stages of the same clusters as at the universities, the SME side is represented by the MT-System Ltd., and it contributes to the dissemination work package, too. The biggest consortia partner, GE Hungary, takes a share in all 
R\&D and demonstration work packages. The Computer and Automation Research Institute, Hungarian Academy of Sciences, as the project leader, takes part in all of the work packages, giving the three clusters' managers, and contributes to the dissemination activities, too.

Over the research results, the main outputs in first cluster are:

- a complete simulation model of a modern production plant,

- $\quad$ solution for production scheduling for the same plant,

- tool for production project scheduling for manufacturing organisation of different structures (Erdélyi, et al., 2002).

The second cluster makes basic research, R\&D activities and demonstrations of realisations for extending the today decision support systems by multimedia and tele-presence features (Haidegger, Popa, 2002).

The paper describes the main targets, steps and results of the third cluster, called "Monitoring of complex production systems" of the above introduced project.

The monitoring field is of special nature because:

- The production process is already fully planned and the main task is to execute the processes as determined. As experienced, the idea being fully planned is impaired continually, due to productand production developments, changes in organisation or economic conditions, etc., which results in a constant re-thinking of certain, usually smaller, manufacturing steps. These are typical changes making it worth to modify the permanent production.

- Changes and disturbances are arising during the production - monitoring has to track and control these disturbances and their effects on the production processes. Several cases showed that these kinds of disturbances can be eliminated from the industrialized environment through appropriate control methods.

- There is a large amount of information about running production processes: machines have several built-in sensors, providing measured signals serving as an important data-source about the current state of manufacturing. This capability makes applying monitoring systems, possible.

- On the one hand, production processes are complex, on the other, disturbances arise continually during production, consequently, the information received by using today's monitoring systems is difficult to analyse. As experienced, the importance of collecting monitoring parameters is usually recognised (stressed, e.g., by today's quality assurance systems, or often by the customer requirements), but sometimes these data are not analysed deeply enough. This unsatisfactory data handling sometimes depreciates the value of the monitoring data and systems applied.

The next tree paragraphs illustrate the results according to the work packages of the cluster:

- Research issues and solutions

- R\&D assignments

- Demonstrations

\section{RESEARCH ISSUES AND SOLUTIONS}

For treating different disturbances and their effects arising during production, monitoring systems collect several measured data coming from several sensors. Through analysing an applied monitoring system, one can recognise that the amount of data information collected in this way is very large. Highlevel of data means a great variety of manufacturing parameters and also a large number of measurements on these parameters. Partially understood relations among these data are one of the characteristics of a supervised production system.

Reliable process models are extremely important in different fields of computer integrated manufacturing (Merchant, M.E., 1998). They are required e.g., for selecting optimal parameters during process planning, for designing and implementing adaptive control systems or model-based monitoring algorithms.

In the CIRP survey on developments and trends in control and monitoring in machining processes, the necessity of sensor integration, sophisticated models, multimodel systems, and learning ability was outlined (Tönshoff et al., 1988). Artificial neural networks (ANNs), neuro-fuzzy (NF) systems are general, multivariable, non-linear estimators, and therefore, they offer a very effective process modelling approach. Soft computing techniques like this seem to be a viable solution on lower level of intelligent, hierarchical control and monitoring systems where abilities for real-time functioning, uncertainty handling, sensor integration, and learning are essential features. These features allow us to apply ANNs, as process models on different levels of production control (Monostori et al., 1996).

Because of the great variety of the monitoring parameters above, it is very difficult to build up a comprehensive model for a production process even if a part of the whole system is modelled. Identifying parts which can be modelled independently, is one of the main issues of modelling. A very important goal of research is to automatically determine such individual parts, based on the monitoring parameters and on artificial neural network models. Fig. 1. shows a resulted sub-model structure having five main parts (in brackets), dividing a system containing eleven (indexed from zero to ten) description parameters. The fourth row of the demonstrated software window, e.g., shows that the algorithm identified a sub-model where parameters $\mathrm{nr}$. 2, 3 and 6 as model inputs are able to estimate the variable $\mathrm{nr}$. 
5. The four identified sub-models have common parameters, e.g. parameter nr. 6 is estimated by the sub-model showed in the second row, but it is to be found among the input variables of the two next submodels, too, showing that this technique recognises a structure of connected sub-models, over the identification of its individual parts.

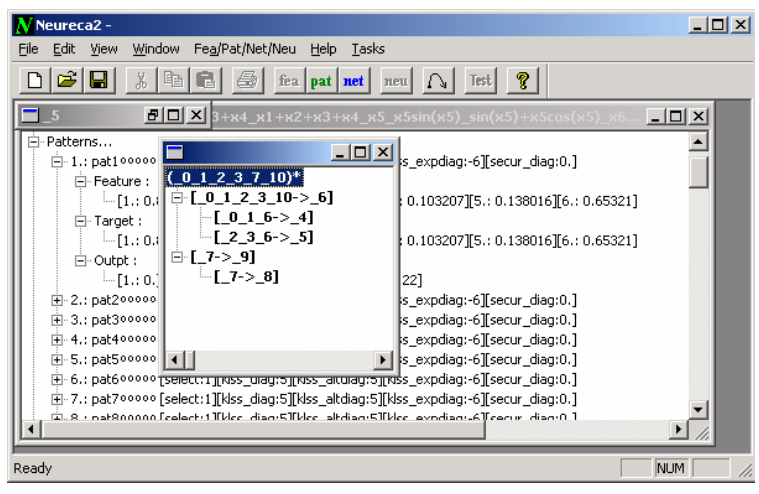

Fig.1.The resulted sub-model structure of a complex system

The large number of measurements on monitoring parameters causes a certain amount of incomplete data, naturally. This fact necessitated making the artificial neural network based process models able to handle incomplete data in their training and application phases, as well.

The expert facing a task recognises that a model is needed to solve it, since some parameters have to be determined based on other ones. Several attempts can be found in the literature to solve various engineering assignments by using neural network based models (Monostori et al., 1996). Several authors use the same concept to select the input and output parameters of the applied neural network, namely, parameters are known in the application phase, are selected as inputs and the model has to estimate the output parameters which are unknown in the assignment. Consequently, the predefined assignment determines the input-output configuration of the model.

A special requirement for modelling is to satisfy the accuracy demands acceptable in certain situations. The requirement cannot always be met in the assignment dependent input-output configuration, i.e., it is not obvious that this setting realises the most appropriate mapping between the variables, consequently, a method finding the appropriate input-output configuration of the applied neural network model is needed. The developed, novel model building process runs totally independently of the assignment (Monostori and Viharos, 2001; Viharos and Monostori, 2002). The aim of the method reviewed here is to decide about all of these parameters whether they should act as inputs or outputs, and to give also the multilayer perceptron model. The method is a modified back-propagation learning algorithm. A flag is used in the developed algorithm to indicate if a neuron of the ANN model is "protected" namely used, or not. (Viharos and Monostori, 1999)

The incomplete data-handling algorithm is based on the main idea of turning the neurons corresponding to the missing part of certain data vectors into protected state and leaving the other neurons in unprotected state (Viharos et al., 2002b). Further information is needed to realise this, i.e., to describe which part of the input- and output vector is missing. A flag called validity is used for indicating whether a data in the data vector is valid or not. It shows that a so-named (binary) validity vector is attached to all of the input and output vectors to describe the validity state of the data incorporated in these vectors. The protection of the input and output layer of the ANN structure changes according to the validity vector of the data vector in question, namely if a date is invalid in the input or output vector, the corresponding neuron is set to be protected, otherwise, the neuron will be unprotected (Monostori and Viharos, 2001).

The developed algorithm was compared to three data-extending-methods and resulted in a model with superior estimation capabilities (Viharos et al., 2002b). The algorithm tested through artificial data was found to be completely able to handle missing output data. Tests showed that it is worth eliminating learning vectors with incomplete input vectors if input parameters are totally independent. An interesting result arose in the case of incomplete input data if input parameters are redundant: it is worth impairing the data purposely in the model building stage to prepare the ANN model for handling incomplete data efficiently in the application phase.

The detailed solution for this assignment is found in (Viharos et al., 2002b).

\section{R\&D ASSIGNMENTS}

The R\&D assignments can be ordered along three main lines:

- Extension of functionalities and datapresentation tools and techniques of today's monitoring systems.

- Earlier, not fully elaborated production monitoring and control assignments are solved through identifying appropriate, sometimes new measurement signals and finding the dependencies among various manufacturing measures applying the intelligent systems described in the previous paragraph.

- Developing a flexible production simulation software tool, which behaves as the real production line and produces monitoring signals just like the physical system. 


\subsection{Extension of functionalities and data- presentation tools and techniques of today's monitoring systems}

A continuous extension of the functionality of applied monitoring systems is an important trend nowadays. The systems have to provide more and more detailed information by using less and less complex presentation tools. Finding appropriate techniques, presenting and highlighting important manufacturing information is among the fundamental goals of the R\&D activities of the project. New, monitoring-oriented data representation charts were defined and implemented satisfying the requirements of the latest monitoring signals, supervisory assignments, engineer aspects and applied quality assurance systems. This implementation entails to develop the appropriate data communication interfaces, storage and retrieval solutions. For preventing the explosion of the industrial databases resulted in size and data handling time, life-length classes of data were defined and ordered to different measured or calculated parameters, regarding manufacturing and organisational performance. The time limit is one of the main features of data lifelength classes, meaning that the data ordered into this class, will be erased in case the time, counted from their collection exceeds.

\subsection{Targeted $R \& D$ on applied modelling of production systems}

The R\&D work started with organising presentations about cluster partners' past results, current work tasks and problems, future directions and targets. This collective review and the derived know-how transfer helped to highlight the main concepts and targets of the common R\&D. After collecting several possible fields of developments and evaluating them based on business requirements, technical opportunities and project frames, a part of them were appointed for the concrete cluster work. The surmountable difficulty in this stage was to step over the cluster members' internal, organisational borders. Having clearly defined R\&D assignments, the concerning workforce and a project-wide work plan was agreed upon for all of the participants. The R\&D activity of the cluster will be completed in the close future. As appear in the next paragraphs, authors have a quite difficult task to explain the details of the performed R\&D assignments coming from the security expectations of the cluster members.

One of the most successful results of the cluster was to develop a system for $100 \%$ testing of the workpieces in aspect to identify a special production failure. $100 \%$ means the observation of all produced pieces of products. As experienced, of this kind of manufacturing problem is among the most frequently occurring failure, consequently, its identification has important financial and quality effects. Nowadays, this type of failure is eliminated through continuous human inspection which yields a quite hard employee work regarding the habituation and stress aspects. Sometimes the evaluation of the same workpiece depends on the person performing it, which shows the possibility for improvement in quality check reliability. On the one hand, people evaluating the work-pieces possess very important know-how, because they can identify this kind of manufacturing problem by a short observation. On the other hand, till now, there has not been any signal known which could be used for the detection. Naturally, after the first project steps we had only some ideas about useful signals, but an appropriate signal was found during the cluster work. A two-level-evaluation and modelling followed the first measurements of the selected signal to prove its applicability. Today, just like in the past, and in our opinion, also in the near future no explicit description for the connection between the values of the measured signal and the production failure will be produced. Still engineers say: "there is "something" in the signal curve". This is a typical case for applying techniques capable to learn, having the possibility to perform several measurements, too. The above ANN-based methods were used to build up the production supervising model. The first level of evaluation proved the applicability of the selected signal and the modelling method. The second level of study moves into the direction of on-line, realized manufacturing equipment. One of the next activities could be to use the resulted production supervising model for production control, as in (Viharos and Monostori, 1999; Viharos et al., 2002a; Viharos et al., 2002b).

Another targeted R\&D work is quite similar to the above, on the one hand. It tries to build up production models where dependencies among parameters are unknown. The modelling is based on learning form collected manufacturing data sets. On the other hand, the task to be solved is quite different from the above. No measurements are needed in this case, because there is a huge number of related monitoring parameter. These data are stored in big databases incorporating the high value of information on the experience through production supervision collected in course of several years. Engineers' opinion is a little bit different from that of in the first case, namely: "it should be some connections among these parameters". The new sub-model finding technique, illustrated in Fig. 2., is applied beyond the ANNbased model building with predetermined inputoutput parameters, for analyzing the connection among data.

\subsection{Monitoring-oriented simulation of production lines}

The development of a monitoring system is a complex assignment; it requires an appropriate collection of relevant demands and a precise description of the related production system, and it will result in a well designed, implemented and reliable monitoring software. These requirements necessitate that the preparation of the physical production equipment should precede the implementation of the monitoring software. 
Consequently, the implementation of a production system - monitoring solution pair requires a long period of time. A software was developed to shorten this development time. It simulates the behaviour of the production equipment and serves with the same information through the same communication protocol as the real production system did. This provides a possibility to build up a model before the preparation of the physical equipment and the development of the monitoring system can be started earlier or parallel with the realization of the physical production system.

After formulating the simulation goals, the first and very important step was to define which part of data information flow, starting from the sensors of machines and ending in company wide databases and presentation tools, is served by the developed software. Data from higher level of information flow, consequently, closer to its end storage and presentation was selected from the two basic possibilities. It was followed by the analysis and behaviour description of members in the production line selected earlier. In contrast to the first impression that this step is easy to overcome, the behaviour exploration and exact description of the system brought up several questions and problems of modelling. This activity resulted in a conceptual description document.

A common decision has to be made, either to use commercial simulation tools or to develop a new software. Knowing the real-time functionality expectations, and evaluating the earlier experience on speed performance and limits of data interaction amount of commercial solutions drove the cluster to build up a new system.

The next document contains the detailed software description preceding the development, including the identification about individual software parts, the related development objects, variables and functions, the appropriate communication protocol, software surface and functionalities, etc. Having the scheduled development plan, the software was implemented through several months. Its name is M2ATRIX representing the basic entities of its internal parts and satisfying marketing aspects, too. Fig. 2. shows the layout of the completed M2ATRIX.

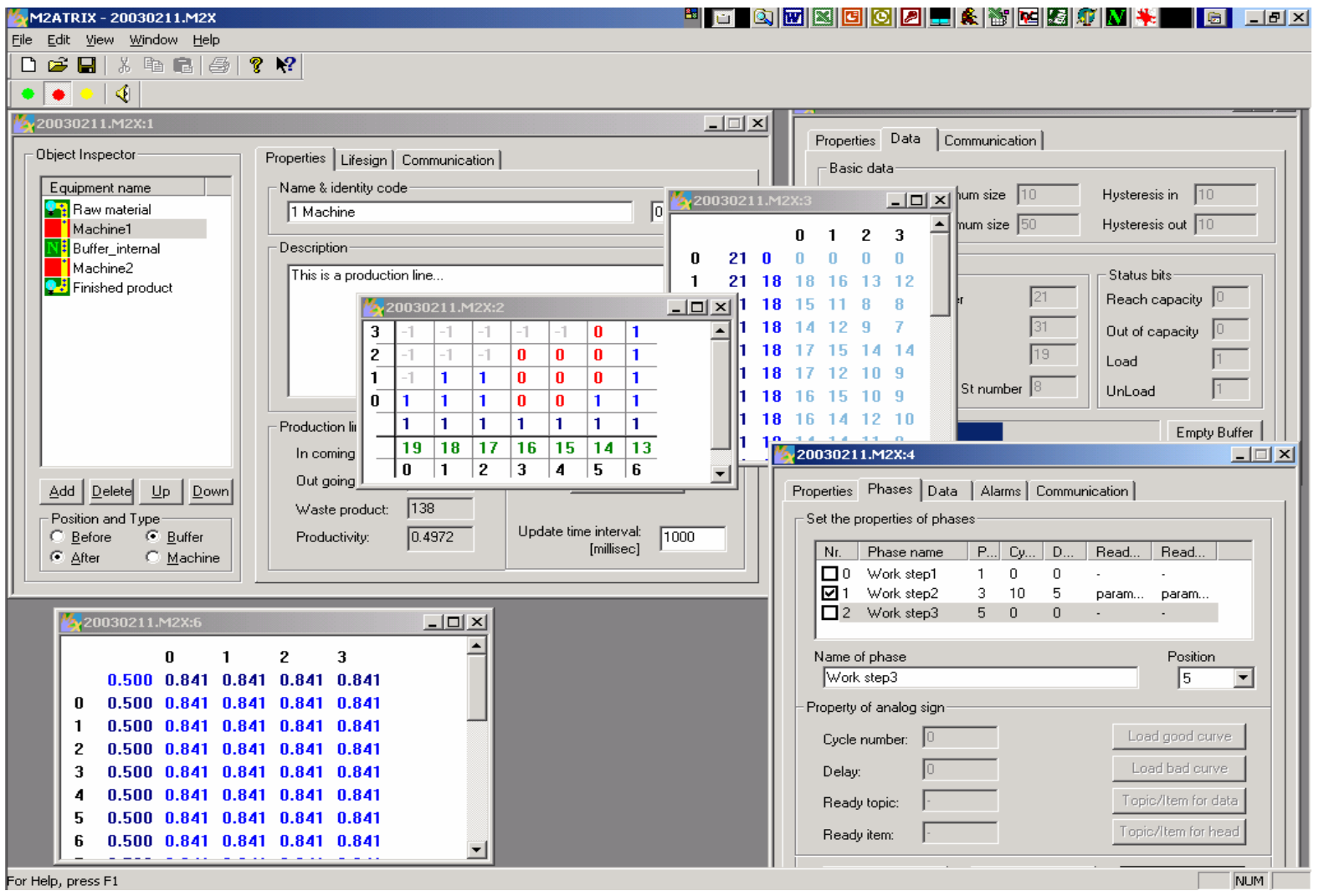

Fig. 2. Software M2ATRIX simulating a small, demo production line 


\section{DEMONSTRATIONS}

The cluster work ends with the demonstration of the solutions realised for:

- modelling and AI based handling of processes and process chains and providing applicable information for the different levels of production control based on sometimes partially incomplete monitoring data,

- improving the information content of the representation of monitoring data,

- simulating the behaviour of the production equipment serving with the same information through the same protocol as the real production system did.

\section{CONCLUSION}

The paper highlights the main targets, solutions and results of the cluster called "Monitoring of complex production systems" of the project on Digital Enterprises, Production Networks supported by the National Research and Development Programme (NRDP) in Hungary. The paragraphs are organised according the comprehensive cluster concept starting with basic and applied research, followed by research and development assignments, and ending in marketoriented demonstrations. The state of the project and also the cluster is right in the middle of the whole working period, some research assignments have been finished (Viharos et al., 2002a; Viharos and Monostori, 1999; Viharos et al., 2002b), the first R\&D results are evaluated and tested, and the demonstrations are just being started.

\section{ACKNOWLEDGEMENT}

The research was supported partially by the project "Digital enterprises, production networks" in the frame of the National Research and Development Programme by the Ministry of Education (proj. No. 2/040/2001) and by János Bolyai Research Fellowship for Dr. Zsolt János Viharos. A part of the work was covered by the National Research Foundation, Hungary, Grant Nos. T043547 and T034632.

\section{REFERENCES}

Erdélyi, F., Tóth, T, Somló, J., Kovács, A., Kádár, B., Márkus, A., Váncza, J. (2002). Production Management: Taking up the challenge of integration. Third Conference on Mechanical Engineering, pp. 705-709.

Haidegger, G., Popa, A. (2002). Assessment of multimedia technologies for use in digital factory projects. Third Conference on Mechanical Engineering, pp. 495-499.

Merchant, M.E. (1998). An interpretive look at 20th century research on modelling of machining. In: Proc. of the CIRP International Workshop on
Modelling of Machining Operations, Inaugural Address, pp. 27-31.

Monostori, L. (2002) Digital enterprises, production networks, Proceedings of the Third Conference on Mechanical Engineering, pp. 540-544.

Monostori, L., Haidegger, G., Váncza, J., Viharos, ZS.J. (2002). Digital Enterprises: A national R\&D project in Hungary, Proceedings of the 1st CIRP (UK) Seminar on Digital Enterprise Technology, DET02, pp. 269-272.

Monostori, L., Márkus, A., Van Brussel, H., Westkämper, E. (1996). Machine learning approaches to manufacturing. Annals of the CIRP, Vol. 45, No. 2, pp. 675-712

Monostori, L., Viharos, Zs. J. (2001). Hybrid, AI- and simulation-supported optimisation of process chains and production plants. Annals of the CIRP, Vol. 50, No. 1, pp. 353-356.

Tönshoff, H.K., Wulsberg, J.P., Kals, H.J.J., König, W., Van Luttervelt, C.A. (1988). Developments and trends in monitoring and control of machining processes. Annals of the CIRP, Vol. 37, No. 2, pp. 611-622

Viharos, Zs. J., Monostori, L. (2002). Optimisation of production systems using simulation and artificial intelligence techniques. Third Conference on Mechanical Engineering, pp. 579583.

Viharos, Zs. J., Monostori, L., Novák, K., Tóth, G.A., Kenderesy, T., Csongrádi, Z., Kórodi, T. (2002a). Monitoring parameter based determination of production tolerances. Third Conference on Mechanical Engineering, pp. 574-578.

Viharos, Zs. J., Monostori; L. (1999). Automatic input-output configuration of ANN-based process models and its application in machining. Lecture Notes of Artificial Intelligence - Multiple Approaches to Intelligent Systems, Springer Computer Science Book, Springer-Verlag Heidelberg, pp. 659-668.

Viharos, Zs.J., Monostori, L., Vincze, T. (2002b). Training and application of artificial neural networks with incomplete data., The Fifteenth International Conference on Industrial \& Engineering Application of Artificial Intelligence \& Expert Systems, Book: Lecture Notes of Artificial Intelligence, Springer Computer Science Book, Springer-Verlag Heidelberg, pp. 649-659. 